\title{
About the Dirichlet boundary value problem using Clifford Algebras
}

\author{
D. Armendádriz ${ }^{\sharp 1}, \quad$ J. Ceballos ${ }^{\dagger}, \quad$ A. Di Teodoro ${ }^{\sharp, 2}$ \\ \# Departamento de Matemáticas, Colegio de Ciencias e Ingenierías \\ Universidad San Francisco de Quito-Ecuador, Diego de Robles y vía Interoceánica \\ E-mail: darmendarizp@estud.usfq.edu.ec ${ }^{1}$ \\ E-mail: nditeodoro@usfq.edu.ec ${ }^{2}$ \\ ${ }^{\dagger}$ Escuela de ciencias físicas y matemáticas. \\ Universidad de Las Américas, Quito-Ecuador Avenida Granados y Colimes. \\ Email: johan.ceballos@udla.edu.ec
}

Dedicated to Professor Tutschke on the occasion of his birthday number 84, Grandmaster. ${ }^{1}$

\begin{abstract}
This paper reviews and summarizes the relevant literature on Dirichlet problems for monogenic functions on classic Clifford Algebras and the Clifford algebras depending on parameters on $\mathbb{R}^{3}$. Furthermore, our aim is to explore the properties when extending the problem to $\mathbb{R}^{n}$ and, illustrating it using the concept of fibres. To do so, we explore ways in which the Dirichlet problem can be written in matrix form, using the elements of a Clifford's base. We introduce an algorithm for finding explicit expressions for monogenic functions for Dirichlet problems using matrices in $\mathbb{R}^{3}$. Finally, we illustrate how to solve an initial value problem related to a fibre.
\end{abstract}

Keywords: Dirichlet problem, Clifford-type algebras, Depending on parameters algebras, Monogenic functions, Cauchy-Riemann operator, Matrix representation.

MSC 2010: 35F15, 30G35.

1 Professor Wolfgang Tutschke (born September 28, 1934 in Görlitz) is a German mathematician. He studied at the University of Leipzig (Ph.D. 1959), was habilitated in 1965 at the Humboldt University in Berlin.

In 1967 he became a professor at the Martin Luther University Halle-Wittenberg. Then in 1992 he became a professor at the University of Technology in Graz. Today the professor is retired, nevertheless he continues teaching, doing research and training doctoral students. The professor Tutschke visited Ecuador in 2014, where he taught a course of hypercomplex analysis. 


\section{Introduction}

\subsubsection{A higher-dimensional commutative structure and its disadvantages}

To define a product of vectors in $\mathbb{R}^{n+1}$ with $n \geq 2$, we may consider ordinary polynomials in $X_{1}, \ldots, X_{n}$, where $X_{j}$ represents the $x_{j}$-axis, $j=1, \ldots, n$. The $x_{0^{-}}$ axis represents the real numbers $x_{0}$. Considering ordinary polynomials in $X_{1}$, $\ldots, X_{n}$ does not imply distinguishing two terms, which are different only in the order of factors $X_{j}$. By identifying squares $X_{j}^{2}$ with real numbers -1 , one obtains a finite-dimensional extension of $\mathbb{R}^{n+1}$ whose elements are finite linear combinations of the $2^{n}$ basis elements $1, X_{1}, \ldots, X_{1} X_{2}, \ldots, X_{1} X_{2}, \cdots X_{n}$. Since the order of the factors is not relevant, we then get an extension of $\mathbb{R}^{n+1}$, where the product is commutative. A disadvantage of this approach is that the product of elements in $\mathbb{R}^{n+1}$ does not remain in $\mathbb{R}^{n+1}$; however, there is no alternative approach to avoid this issue.

Moreover, in this extension the Laplace operator cannot be factorized in its two first order operators. If $\partial_{\bar{z}}$ is the differential operator $\frac{1}{2}\left(\partial_{x}+i \partial_{y}\right)$ and $\partial_{z}$, its conjugate, is defined by $\partial_{z}=\frac{1}{2}\left(\partial_{x}-i \partial_{y}\right)$, then:

$$
4 \partial_{z} \partial_{\bar{z}}=d_{2} \text {, }
$$

Thus, the Laplace operator $d_{2}$ in the plane can be factorized by the two first order differential operators. Since such factorizations are useful for many applications, it will be important to generalize $D$ from the Cauchy-Riemann operator by factorizing the Laplace operator in $\mathbb{R}^{\mathbf{n}+1}$.

The construction above, which has a commutative product, does not have this property. First, observe that the rules $X_{j}^{2}=-1$ and $X_{i} X_{j}=X_{j} X_{i}$ lead to a $2^{n}$ dimensional extension of $\mathbb{R}^{n+1}$ having the usual basis

$$
\beta_{n}=\left\{1, e_{1}, \ldots, e_{n}, e_{1} e_{2}, \ldots, e_{n-1, n}, \ldots, e_{1} e_{2} \cdots e_{n}\right\}
$$

with the identification $X_{j}=e_{j}$ for $j \in\{1,2, \ldots, n\}$. See $[2,3]$.

The functions $u=u(x)$ take values in the Algebra constructed above, that is, $u(x)$ can be written as $\sum_{A} u_{A}(x) e_{A}$, where $u_{A}(x)$ are real-valued components of $u(x)$ and $e_{A} \in \beta_{n}$. While holomorphic functions $w=w(z)$ in the $z$-plane are defined by $\partial_{\bar{z}} w=0$, in higher dimensions it is necessary to define an analogous class of functions $u=u(x), D u=0$, where $D$ is the generalized CauchyRiemann operator defined by

$$
D=\sum_{i=0}^{n} e_{i} \partial_{j}
$$

where $\partial_{j}$ represents the derivative with respect to $x_{j}$. The conjugate CauchyRiemann operator $\bar{D}$ to $D$ is given by

$$
\bar{D}=\partial_{0}-\sum_{j=1}^{n} e_{j} \partial_{j} .
$$


Since $e_{i}^{2}=-1$ and $e_{i} e_{j}=e_{j} e_{i}$, we get

$$
\begin{aligned}
\bar{D} D & =\left(\partial_{0}-\sum_{i=1}^{n} e_{i} \partial_{i}\right)\left(\partial_{0}+\sum_{j=1}^{n} e_{j} \partial_{j}\right) \\
& =\partial_{0}^{2}+\sum_{i=1}^{n} \partial_{i}^{2}-\sum_{i \neq j} e_{i} e_{j} \partial_{i} \partial_{j} \\
& =\Delta_{n+1}-\sum_{i<j}\left(e_{i} e_{j}+e_{j} e_{i}\right) \partial_{i} \partial_{j} \\
& =\Delta_{n+1}-2 \sum_{i<j} e_{i} e_{j} \partial_{i} \partial_{j},
\end{aligned}
$$

where $\Delta_{n+1}$ is the Laplace operator in $\mathbb{R}^{\mathbf{n}+\mathbf{1}}$. Given that mixed derivatives appear in the latter formula, the Laplace operator cannot be factorized by the first order operators $D$ and $\bar{D}$. Moreover, since not all coefficients in (3) are real, the equation $\bar{D} D u=0$ is a coupled system for the real-valued components $u_{A}$ with solutions $u=\sum_{A} u_{A} e_{A}$ of $D u=0$. See $[6,8]$.

Thus, we can now ask ourselves which structure is more useful for factorizing the Laplace operator. This is our motivation for the introduction of Clifford algebras

\subsubsection{The usual Clifford algebra defined by equivalence classes}

The starting point for the study of Clifford algebras are bilinear forms in linear spaces (see the basic monography [6] from F. Brackx, R. Delanghe and F. Sommen). However, the usual Clifford Algebras over $\mathbb{R}^{n+1}$ can also be constructed as equivalence classes of the $n$ free independent variables $X_{1}, \ldots, X_{n}$, where one has to distinguish the two terms $X_{\mu_{1}} X_{\mu_{2}} \cdots X_{\mu_{m}}$ with factors that are in different order.

Equation (3) shows that it is possible to get a factorization of the Laplace operator provided $e_{i} e_{j}+e_{j} e_{i}=0$ for each pair $i \neq j$. Thus, a Clifford Algebra leading to the factorization $\Delta_{n+1}=\bar{D} D$ can be obtained if the formal structure is

$$
X_{j}^{2}+1 \quad \text { and } \quad X_{i} X_{j}+X_{j} X_{i}
$$

where $i, j=1, \ldots, n$ and $i \neq j$.

We now are in a position to formally define Clifford Algebras. Consider $n$ variables $X_{1}, \ldots, X_{n}$ in a free algebra or free $\mathcal{R}$-module, where the two products

$$
X_{\mu_{1}} \cdots X_{\mu_{m}}
$$

are to be distinguished if the order of the factors is different (For instance, $X_{1} X_{2} X_{3}$ is said to be different from $X_{2} X_{1} X_{3}$ ). 
Definition Two formal combinations, $P$ and $Q$ on $\mathcal{R}\left[X_{1}, \ldots, X_{n}\right]$, are said to be equivalent if in their difference we can see one of the following terms:

$$
X_{j}^{2}+1, \quad j=1, \ldots, n,
$$

or

$$
X_{j} X_{k}+X_{k} X_{j}, \quad j \neq k .
$$

When two formal combinations, $P$ and $Q$, are equivalent, we write $P \sim Q$.

Definition The Clifford Algebra $\mathcal{A}_{n}$ is the set of all equivalence classes of formal combinations in $\mathcal{R}\left[X_{1}, \ldots, X_{n}\right]$ with respect to the equivalence relation $\sim$.

Observe that in particular we have

$$
X_{j}^{2}+1 \sim 0 \quad \text { and } \quad X_{j} X_{k}+X_{k} X_{j} \sim 0 \quad(j \neq k) .
$$

This means, in the language of equivalence classes, that

$$
X_{j}^{2}+1=0 \quad \text { and } \quad X_{j} X_{k}+X_{k} X_{j}=0 \quad(j \neq k) .
$$

This, however, is the same as

$$
X_{j}^{2}=-1 \quad \text { and } \quad X_{j} X_{k}=-X_{k} X_{j} \quad(j \neq k) .
$$

\section{Remark 1}

- Algebraic properties (such as the associativity of the algebraic operations and the distributive law) follow from the corresponding properties of polynomials. Note, however, that the multiplication is not commutative because $X_{j} X_{k}=$ $-X_{k} X_{j}$ for $j \neq k$.

- The relations in (4) are called structure relations of the Clifford Algebra. See $[28,29]$

- $\mathcal{A}_{n}$ is a linear space of dimension

$$
1+n+\left(\begin{array}{l}
n \\
2
\end{array}\right)+\cdots+\left(\begin{array}{c}
n \\
n-1
\end{array}\right)+1=(1+1)^{n}=2^{n} .
$$

Example 1 In $\mathcal{A}_{2}$ the structure relation is given by

$$
\left\{\begin{array}{l}
e_{0}=1 \\
e_{i}^{2}=-1 \quad \text { where } i=1,2 \\
e_{1} e_{2}+e_{2} e_{1}=0
\end{array}\right.
$$

The basis is

$$
\left\{e_{0}=1, e_{1}, e_{2}, e_{12}\right\} .
$$

An element different from 0 is given by $a=a_{0}+a_{1} e_{1}+a_{2} e_{2}+a_{12} e_{12}$. 
Recently, Clifford analysis associated to new algebraic structures has attracted special attention. If one generalizes the structure used to define the Clifford Algebra, one obtains non-classical Clifford Algebra. Even though, classical Clifford Algebras have been sufficiently developed in the last decade, there is a need to study properties resulting from a more general structure, which allow for a better understanding of natural phenomena. $[5,9,12,18-20$, 22]

\subsubsection{Clifford Algebras depending on parameters}

Let $p$ be any parameter running on certain set and, let $\alpha_{j}(p)$ and $\gamma_{i j}(p)=\gamma_{j i}$ be real-valued functions depending on that parameter $p$, where $i, j=1, \ldots, n$ and $i \neq j$. Furthermore, $k_{j} \geq 2$ are natural numbers. For applications to partial differential equations in a domain $\Omega$ of $\mathbb{R}^{n+1}$, one may assume that $p$ is the variable $x$ running in $\Omega$.

Consider $n$ free independent variables $X_{1}, X_{2}, \ldots, X_{n}$ over $\mathbb{R}^{n}$. Then, the vectors of $\mathbb{R}^{\mathbf{n}+\mathbf{1}}$ can be represented with a linear combination of the $n$ free independent variables, where this identification preserves the linear structure. As before, one has to distinguish the two terms $X_{\mu_{1}} X_{\mu_{2}} \cdots X_{\mu_{m}}$ with factors that are in different order.

That way, one gets an infinite-dimensional extension of $\mathbb{R}^{n+1}$ in which, the product of vectors can be computed. In order to obtain only a finite-dimensional extension, we consider a equivalence class through the structures

$$
X_{j}^{k_{j}}+\alpha_{j}(p) \text { and } X_{i} X_{j}+X_{j} X_{i}-2 \gamma_{i j}(p),
$$

where $i, j=1, \ldots, n$ and $i \neq j$.

Using this structure, each term of a formal linear combination of $X_{1}, \ldots, X_{n}$ can be written as

$$
c X_{1}^{v_{1}} \cdots X_{n}^{v_{n}},
$$

where $c$ is a real constant and the exponents $v_{j}$ are not greater than $k_{j}-1$, i.e., $0 \leq v_{j} \leq k_{j}-1$.

The Clifford algebra generated by the structure (5) will be denoted by

$$
\begin{aligned}
& \mathcal{A}_{n}\left(p \mid k_{j}, \alpha_{j}(p), \gamma_{i j}(p)\right) \text { if } n \geq 2, \quad \text { and } \\
& \mathcal{A}_{1}(p \mid k, \alpha(p)) \text { if } n=1 .
\end{aligned}
$$

When the coefficients $\alpha_{j}, \gamma_{i j}$ do not depend on the parameter $p$, we write

$$
\mathcal{A}_{n}\left(k_{j}, \alpha_{j}, \gamma_{i j}\right) \text { and } \mathcal{A}_{1}(k, \alpha) \text { resp. }
$$

instead of (6). As usual, one denotes $X_{j}$ by $e_{j}, e_{1} e_{2}$ by $e_{12}$ and, so on. Then, for $\mathcal{A}_{n}\left(p \mid k_{j}, \alpha_{j}(p), \gamma_{i j}(p)\right)$ has the basis

$$
e_{1}^{v_{1}} e_{2}^{v_{2}} \cdots e_{n}^{v_{n}}, \quad 0 \leq v_{j} \leq k_{j}, \quad j=1, \ldots, n,
$$


and so we have,

$$
\operatorname{dim} \mathcal{A}_{n}\left(p \mid k_{j}, \alpha_{j}(p), \gamma_{i j}(p)\right)=k_{1} \cdots k_{n}
$$

for $n \geq 2$ and for $n=1$

$$
\operatorname{dim} \mathcal{A}_{1}(p \mid k, \alpha(p))=k \text {. }
$$

For instance, $\mathcal{A}_{2}(3,1,0)$ is 9 -dimensional and, has basis:

$$
1, e_{1}, e_{2}, e_{1}^{2}, e_{1} e_{2}, e_{2}^{2}, e_{1}^{2} e_{2}, e_{1} e_{2}^{2}, e_{1}^{2} e_{2}^{2} .
$$

Furthermore, $\mathcal{A}_{2}\left(k_{j}, 1,0\right)$ with $k_{1}=2$ and $k_{2}=3$ has dimension 6 and, its basis is

$$
1, e_{1}, e_{2}, e_{1} e_{2}, e_{2}^{2}, e_{1} e_{2}^{2} \text {. }
$$

The usual Clifford Algebra is $\mathcal{A}_{n}(2,1,0)$. The basis for the Clifford Algebra $\mathcal{A}_{1}(4,1)$ is $1, e_{1}, e_{2}=e_{1}^{2}$ and $e_{3}=e_{1}^{3}$ and, so its dimension is 4 . The dimension for the Clifford Algebra $\mathcal{A}_{n}\left(p \mid 2, \alpha_{j}(p), \gamma_{i j}(p)\right)$ for $n \geq 2$ is $2^{n}$. And, it has the usual basis $1, e_{1}, \ldots, e_{n}, e_{12}, \ldots, e_{12 \ldots n}$.

Finally, one important difference between classical Clifford Algebras and the Clifford-type Algebras is the second-order differential operator:

$$
\tilde{\Delta}=\bar{D} D=\partial_{0}^{2}+\sum_{j=1}^{n} \alpha_{j} \partial_{j}^{2}-2 \sum_{i<j} \gamma_{i j} \partial_{i} \partial_{j}
$$

See the papers $[22,29,30]$ for more details.

\subsubsection{Clifford valued function}

Let $\Omega$ be an open and connected domain in $\mathbb{R}^{n+1}$ with points $x=\left(x_{0}, x_{1}, \ldots, x_{n}\right)$. Furthermore, let $u(x)$ be a function with values in $\mathcal{A}_{n}\left(2, \alpha_{j}, \gamma_{i j}\right)$ defined on $\Omega$. Denoting the real-valued components of $u(x)$ by $u_{A}(x)$, that is,

$$
u(x)=\sum_{A} u_{A}(x) e_{A}
$$

Where $A$ is a set of index combination. See $[4,6,8,17,29]$.

Definition 1 We say that $u(x)$ is a left-monogenic function if the equation $D u=$ 0 is satisfied. If $u(x)$ satisfies the equation $u D=0$, the function is said to be rightmonogenic.

Any Clifford-algebra-valued function $u=\sum_{A} u_{A} e_{A}$, which is monogenic with respect to the usual Cauchy-Riemann operator $D$ and, the Clifford Algebra 
$\mathcal{A}_{n}\left(2, \alpha_{j}, \gamma_{i j}\right)$ introduced above will lead to the homogeneous second-order differential equation:

$$
\begin{aligned}
\bar{D} D u & =\bar{D} D\left(\sum_{A} u_{A} e_{A}\right) \\
& =\partial_{0}^{2} u+\sum_{j=1}^{n} \alpha_{j} \partial_{j}^{2} u-2 \sum_{i<j} \gamma_{i j} \partial_{i} \partial_{j} u \\
& =0 .
\end{aligned}
$$

Remark 2 Hereinafter, we will use $\mathcal{A}_{n, 2}=\mathcal{A}_{n}\left(2, \alpha_{j}, \gamma_{i j}\right)$ to refer to the case $\alpha_{j}, \gamma_{i j}$ constants and $\mathcal{A}_{n, 2}^{*}=\mathcal{A}_{n}\left(2, \alpha_{j}, \gamma_{i j}\right)$ to refer to no constant case.

\section{Dirichlet Boundary value problem}

\subsection{Prescription of the values on the boundary}

Let $z=u+i v$ where $u, v: \mathbb{R}^{2} \rightarrow \mathbb{R}$. The imaginary part for a holomorphic function, $v$, with a bounded domain $\Omega$ in the complex plain is uniquely determined by its boundary values. Then, the Cauchy-Riemann system gives the real part, $u$, for the first order system, which in turn is completely integrable:

$$
\partial_{x} u=\partial_{y} v \text { and } \partial_{y} u=-\partial_{x} v
$$

Therefore, $u$ is uniquely determined in simply connected domains up to a real constant. Thus, $u$ is uniquely determined at any point on $\Omega$ or $\bar{\Omega}$, if applicable. The system for $u$ is completely integrable because $u$ is a solution for the Laplace equation.

Akin to the complex case, a monogenic function, $u$, is continuously differentiable in an open connected set, $\Omega \subset \mathbb{R}^{n+1}$. The function's values belong to a Clifford Algebra $\mathcal{A}_{n}$, such that its $2^{n}$ real-valued components are related through the Cauchy-Riemann system in $\mathbb{R}^{n+1}$, i.e. $D u=0$, consisting of $2^{n}$ real first order partial differential equations in $\Omega$. This condition again implies that it is not possible to arbitrarily and simultaneously prescribe boundary values for all the components. See $[13,29,31]$.

Below, we will present a method to solve the Dirichlet boundary problem in a cylindrical domain in $\mathcal{A}_{2,2}$ and $\mathcal{A}_{2,2}^{*}$. Afterwards, we will present a way to extend the solution in $\mathcal{A}_{n, 2}^{*}$ using distinguished domains.

\subsubsection{Cylindrical domain}

Let $\Omega$ be a cylindrical domain whose closure $\bar{\Omega}$ is given by

$$
\Omega=\left\{x=\left(x_{0}, x_{1}, x_{2}\right): \psi_{1}\left(x_{1}, x_{2}\right) \leq x_{0} \leq \psi_{2}\left(x_{1}, x_{2}\right),\left(x_{1}, x_{2}\right) \in \Omega_{0}\right\},
$$


where $\Omega_{0}$ is a simply connected domain in the $x_{1}-, x_{2}$-plane and, $\psi_{1}$ and $\psi_{2}$ are continuously differentiable in $\overline{\Omega_{0}}$. Then, the lower covering surface of $\Omega$ in the direction of the $x_{0}$-axis is

$$
S_{0}=\left\{x=\left(x_{0}, x_{1}, x_{2}\right): x_{0}=\psi_{1}\left(x_{1}, x_{2}\right),\left(x_{1}, x_{2}\right) \in \overline{\Omega_{0}}\right\} .
$$

Theorem 1 The Dirichlet problem in $\Omega$ for a twice-continuous monogenic function with values in $\mathcal{A}_{2,2}$ is uniquely solvable, where the Dirichlet problem can be described as:

Find a monogenic function with respect to the Clifford Algebra $\mathcal{A}_{2,2}$, for which two components $u_{1}$ and $u_{2}$ are arbitrarily prescribed on the whole boundary $\partial \Omega$, whereas $u_{12}$ has prescribed values on the lower base $S_{0}$ of the cylindrical domain $\Omega$ and $u_{0}$ is prescribed at only one point of $\Omega$.

In $[24,25,29]$, the authors solved a Dirichlet problem in a cylindrical domain for monogenic functions with values in $\mathcal{A}_{2,2}$.

\subsection{Dirichlet boundary valued problem on $\mathcal{A}_{2,2}$ and $\mathcal{A}_{2,2}^{*}$}

\subsubsection{Cauchy-Riemann systems with constant coefficients $(\alpha, \beta, \gamma), \mathcal{A}_{2,2}$ case}

In this case, the differential second order equation

$$
\partial_{0}^{2} u+\alpha \partial_{1}^{2} u+\beta \partial_{2}^{2} u-2 \gamma \partial_{1} \partial_{2} u=0 .
$$

is elliptic if $\gamma^{2}<\alpha \beta$. The Cauchy-Riemann equation $D u=0$ for a monogenic function $u$ in $\mathcal{A}_{2}(2, \alpha, \beta, \gamma)$ is equivalent to the system

$$
\begin{aligned}
& \partial_{0} u_{0}-\alpha \partial_{1} u_{1}+2 \gamma \partial_{2} u_{1}-\beta \partial_{2} u_{2}=0, \\
& \partial_{0} u_{1}+\partial_{1} u_{0}+\beta \partial_{2} u_{12}=0, \\
& \partial_{0} u_{2}-\alpha \partial_{1} u_{12}+\partial_{2} u_{0}+2 \gamma \partial_{2} u_{12}=0, \\
& \partial_{0} u_{12}+\partial_{1} u_{2}-\partial_{2} u_{1}=0,
\end{aligned}
$$

for its four real-valued components $u_{0}, u_{1}, u_{2}$ and $u_{12}$. Note that the parameters $\alpha, \beta$ and $\gamma$ may depend on another variable, for example, $x$ in $\mathbb{R}^{3}$.

Since the four components $u_{0}, u_{1}, u_{2}$ and $u_{12}$ of a monogenic function are solutions for the Laplace equation, $u_{1}$ and $u_{2}$ are uniquely determined by their boundary values for the entire boundary $\partial \Omega$. It follows from equation (13) that knowing $u_{1}$ and $u_{2}, u_{12}$ can be determined by integrating over the $x_{0}$-direction, provided that the values for $u_{12}$ on $S_{0}$ are known (see the graphic illustration ??).

Finally, one can use the remaining three equations (10)-(12) to calculate the component $u_{0}$, splitting up the Cauchy-Riemann system in $\mathbb{R}^{3}$ into two completely integrable first order systems for $u_{12}$ and $u_{0}$, respectively. The component $u_{0}$ can be found from the system 


$$
\begin{aligned}
& \partial_{0} u_{0}=\alpha \partial_{1} u_{1}-2 \gamma \partial_{2} u_{1}+\beta \partial_{2} u_{2}=p_{0}, \\
& \partial_{1} u_{0}=-\partial_{0} u_{1}-\beta \partial_{2} u_{12}=p_{1}, \\
& \partial_{2} u_{0}=-\partial_{0} u_{2}+\alpha \partial_{1} u_{12}-2 \gamma \partial_{2} u_{12}=p_{2} .
\end{aligned}
$$

Since $u_{1}, u_{2}$ and $u_{12}$ are solutions for the Laplace equation, the latter system for $u_{0}$ turns out to be completely integrable, that is $\partial_{k} p_{j}=\partial_{j} p_{k}, k, j=0,1,2$. Provided $\Omega$ is homotopically simply connected, $u_{0}$ is already uniquely determined by its value at one point $P_{0}$ of $\bar{\Omega}$. For more details on the solution, see [30].

Because $\alpha, \beta$ and $\gamma$ may depend on the space-like component, another interesting case is when $\alpha, \beta$ and $\gamma$ depend on $x \in \mathbb{R}^{3}$.

\subsubsection{Cauchy-Riemann systems with non-constant coefficients $(\alpha, \beta, \gamma), \mathcal{A}_{2,2}^{*}$ case}

Now suppose that the parameters $\alpha, \beta$ and $\gamma$ of the structured relations depend on the variable $x$. Then, one obtains the same system (10) - (13) for the components of a monogenic function, but now the coefficients depend on $x$. Then, the differential equations (9) for the components $u_{0}, u_{1}, u_{2}$ and $u_{12}$ are coupled, whereas they are uncoupled when the coefficients are constant. The main parts are always the same, namely

$$
\partial_{0}^{2} u_{j}+\alpha \partial_{1}^{2} u_{j}+\beta \partial_{2}^{2} u_{j}-2 \gamma \partial_{1} \partial_{2} u_{j}, \quad j=0,1,2,12 .
$$

In addition to the principal parts, the second order differential equations for $u_{12}, u_{1}$ and $u_{2}$ have the following linear terms, respectively:

$$
\begin{array}{r}
+\partial_{1} \alpha \cdot \partial_{1} u_{12}-2 \partial_{1} \gamma \cdot \partial_{2} u_{12}+\partial_{2} \beta \cdot \partial_{2} u_{12} \\
+\partial_{0} \beta \cdot \partial_{2} u_{12}+\partial_{1} \alpha \cdot \partial_{1} u_{1}-2 \partial_{1} \gamma \cdot \partial_{2} u_{1}+ \\
\partial_{1} \beta \cdot \partial_{2} u_{2} \\
-\partial_{0} \alpha \cdot \partial_{1} u_{12}+2 \partial_{0} \gamma \cdot \partial_{2} u_{12}+\partial_{2} \alpha \cdot \partial_{1} u_{1}- \\
2 \partial_{2} \gamma \cdot \partial_{2} u_{1}+\partial_{2} \beta \cdot \partial_{2} u_{2} .
\end{array}
$$

A similar expression can be obtained for the differential equation of $u_{0}$.

The differential equation for $u_{12}$ is always uncoupled because the additional linear terms contain only derivatives for $u_{12}$. The equations for $u_{1}$ and $u_{2}$ are coupled only with one another, when the parameters $\alpha, \beta$ and $\gamma$ do not depend on $x_{0}$. The equations for $u_{1}$ and $u_{2}$ are completely uncoupled if $\alpha$ and $\gamma$ depend only on $x_{1}$ and $\beta$ depends only on $x_{2}$.

A similar calculation shows that the system $\partial_{k} p_{j}=\partial_{j} p_{k}, k, j=0,1,2$ is also compatible for the case of non-constant coefficients. If the differential equations for $u_{1}$ and $u_{2}$ are uncoupled, then theorem 1 is also true for non-constant coefficients. See [30]. 
The following step is to solve the Dirichlet boundary value problem in $\mathbb{R}^{n+1}$. In order to generalize our method to a $\Omega \subset \mathbb{R}^{n+1}$, professor Tutschke suggested a generalization of the cylindrical domain, the distinguishing domain. The key idea is that a given domain $\Omega$ can be decomposed into fibres with small parts with certain properties.

\subsection{Decomposition into $\mu$-dimensional fibres}

Consider the Euclidean space $\mathbb{R}^{n+1}$ with the coordinates $x_{0}, x_{1}, \ldots, x_{n}$. Choose $\mu$ with $1 \leq \mu \leq n+1$ and, choose $\mu$ indices $k_{1}, \ldots, k_{\mu}$. Then, the intersections of a given (bounded) domain $\Omega$ with $\mu$-dimensional planes of $\mathbb{R}^{n+1}$ is given by:

$$
\Pi=\left\{x=\left(x_{0}, \ldots, x_{n}\right): x_{k_{1}}=c_{1}, \ldots, x_{k_{\mu}}=c_{\mu}\right\}
$$

Definition 2 We say that the $\mu$-dimensional fibres in the $k_{1}, \ldots, k_{\mu}$-direction of the given bounded domain $\Omega$ are the intersections of the $\Omega$ with $\mu$-dimensional planes.

Definition 3 We say that $\Omega$ can be decomposed into $\mu$-dimensional fibres in $k_{1}$, ..., $k_{\mu}$-direction if there exists a $(1+n-\mu)$-dimensional part $S_{k_{1} \ldots k_{\mu}}$ of $\partial \Omega$ having the following properties:

- There exists a 1-1-projection of $S_{k_{1} \ldots k_{\mu}}$ into a $(1+n-\mu)$-dimensional subspace of $\mathbb{R}^{\mathbf{n}+\mathbf{1}}$.

- In each plane $P$, there exists at most one point of $S_{k_{1} \ldots k_{\mu}}$, which is a boundary point for the corresponding fibre.

- $\bar{\Omega}$ is the product of $S_{k_{1} \ldots k_{\mu}}$ with the closure of the fibres.

The subset $S_{k_{1} \ldots k_{\mu}}$ is called the distinguishing part of the corresponding decomposition of $\Omega$.

Example 2 Consider the cylindrical domain $\Omega \subset \mathbb{R}^{3}$ given in (??). $\Omega$ can be decomposed in the lower or upper covering surface, this is a distinguishing part for the decomposition in the $x_{0}$-direction that represents the 1-dimensional fibre. See [26] for more details on fibres.

Remark 3 Using more general operators of Cauchy-Riemann type, the Dirichlet problem in the cylinder can also be solved, see $[9,11]$.

In the following section, we are going to represent the Cauchy-Riemann system in matrix form in $\mathcal{A}_{2,2}^{*}$, we will also discuss the matrix representation in $\mathcal{A}_{n}$ and, show an example using a computer implementation for $\mathcal{A}_{3}$. See [7]. 


\section{Cauchy-Riemann System in matrix form}

Consider a matrix basis

$$
\beta_{n}=\left\{\mathbf{I}_{2^{\mathrm{n}}}, E_{1, n}, \ldots, E_{n, n}, E_{12, n}, \ldots E_{1 n, n}, \ldots, E_{1, n}\right\}
$$

$\left(2^{n}\right.$-dimensional) for real Clifford Algebras, composed by the matrices $\mathbb{R}^{2^{n} \times 2^{n}}$, with real elements.

These $E_{i, n}, i=1,2, \ldots, n$ satisfy the structure relations:

$$
\begin{aligned}
E_{j, n}^{2} & =-\alpha_{j} \mathbf{I}_{2^{\mathrm{n}}}, \quad \alpha_{j}>0, \\
E_{i, n} E_{j, n}+E_{j, n} E_{i, n} & =2 \gamma_{i j} \mathbf{I}_{2^{\mathrm{n}}}, \quad i \neq j,
\end{aligned}
$$

for $i, j=1,2, \ldots, n$.

Remark 4 Writing the elements for a Clifford Algebra $\mathbb{R}_{0, n}^{*}$ in matrix form facilitates the manipulation of considerable amounts of information, given that all the required data is contained in such matrices.

In our case, the matrix representation and the computer implementation give us an efficient tool to compute the explicit form for the Cauchy-Riemann system and to prove the compatibility conditions.

\section{1 $\mathcal{A}_{2}$ case}

Let $\beta_{2}=\left\{\mathbf{I}_{4}, E_{1,2}, E_{2,2}, E_{12,2}\right\}$ the matrix basis for $\mathcal{A}_{2}$, where:

$$
E_{1,2}=\left(\begin{array}{cccc}
0 & -\alpha_{1} & 0 & 0 \\
1 & 0 & 0 & 0 \\
0 & 0 & 0 & -\alpha_{1} \\
0 & 0 & 1 & 0
\end{array}\right), \quad E_{2,2}=\left(\begin{array}{cccc}
0 & 2 \gamma_{12} & -\alpha_{2} & 0 \\
0 & 0 & 0 & \alpha_{2} \\
1 & 0 & 0 & 2 \gamma_{12} \\
0 & -1 & 0 & 0
\end{array}\right)
$$

and

$$
E_{12,2}=\left(\begin{array}{cccc}
0 & 0 & 0 & -\alpha_{1} \alpha_{2} \\
0 & 2 \gamma_{12} & -\alpha_{2} & 0 \\
0 & \alpha_{1} & 0 & 0 \\
1 & 0 & 0 & 2 \gamma_{12}
\end{array}\right)
$$

and $\mathbf{I}_{4}$ is the identity. Thus a function

$$
u: \mathbb{R}^{3} \rightarrow \mathcal{A}_{2}
$$

can be define by: 


$$
\begin{aligned}
& u(x, y, z)=u_{0} \mathbf{I}_{4}+u_{1} E_{1,2}+u_{2} E_{2,2}+u_{12} E_{12,2} \\
& =\left(\begin{array}{cccc}
u_{0} & 0 & 0 & 0 \\
0 & u_{0} & 0 & 0 \\
0 & 0 & u_{0} & 0 \\
0 & 0 & 0 & u_{0}
\end{array}\right)+\left(\begin{array}{cccc}
0 & -\alpha_{1} u_{1} & 0 & 0 \\
u_{1} & 0 & 0 & 0 \\
0 & 0 & 0 & -\alpha_{1} u_{1} \\
0 & 0 & u_{1} & 0
\end{array}\right)+ \\
& \left(\begin{array}{cccc}
0 & 2 \gamma_{12} u_{2} & -\alpha_{2} u_{2} & 0 \\
0 & 0 & 0 & \alpha_{2} u_{2} \\
u_{2} & 0 & 0 & 2 \gamma_{12} u_{2} \\
0 & -u_{2} & 0 & 0
\end{array}\right)+\left(\begin{array}{cccc}
0 & 0 & 0 & -\alpha_{1} \alpha_{2} u_{12} \\
0 & 2 \gamma_{12} u_{12} & -\alpha_{2} u_{12} & 0 \\
0 & \alpha_{1} u_{12} & 0 & 0 \\
u_{12} & 0 & 0 & 2 \gamma_{12} u_{12}
\end{array}\right) \\
& =\left(\begin{array}{cccc}
u_{0} & -\alpha_{1} u_{1}+2 \gamma_{12} u_{2} & -\alpha_{2} u_{2} & -\alpha_{1} \alpha_{2} u_{12} \\
u_{1} & u_{0}+2 \gamma_{12} u_{12} & -\alpha_{2} u_{12} & \alpha_{2} u_{2} \\
u_{2} & \alpha_{1} u_{12} & u_{0} & -\alpha_{1} u_{1}+2 \gamma_{12} u_{2} \\
u_{12} & -u_{2} & u_{1} & u_{0}+2 \gamma_{12} u_{12}
\end{array}\right)
\end{aligned}
$$

where, $u_{0}, u_{1}, u_{2}, u_{12}: \mathbb{R}^{3} \rightarrow \mathbb{R}$.

The Cauchy-Riemann operator is given by:

$$
\begin{aligned}
& \mathcal{D}_{2}= \\
& \left(\begin{array}{cccc}
0 & -\alpha_{1} \partial_{1}+2 \gamma_{12} \partial_{2} & -\alpha_{2} \partial_{2} & 0 \\
\partial_{1} & 0 & 0 & \alpha_{2} \partial_{2} \\
\partial_{2} & 0 & 0 & 2 \gamma_{12} \partial_{2}-\alpha_{1} \partial_{1} \\
0 & -\partial_{2} & \partial_{1} & 0
\end{array}\right) \\
& =E_{1,2} \partial_{1}+E_{2,2} \partial_{2} .
\end{aligned}
$$

Thus, the Cauchy-Riemann system is given by:

$$
\begin{aligned}
& \mathcal{D}_{2} u \\
& =\left(E_{1,2} \partial_{1}+E_{2,2} \partial_{2}\right)\left(u_{0} \mathbf{I}_{4}+u_{1} E_{1,2}+u_{2} E_{2,2}+u_{12} E_{12,2}\right) \\
& =\left(\begin{array}{cccc}
0 & -\alpha_{1} \partial_{1}+2 \gamma_{12} \partial_{2} & -\alpha_{2} \partial_{2} & 0 \\
\partial_{1} & 0 & 0 & \alpha_{2} \partial_{2} \\
\partial_{2} & 0 & 0 & 2 \gamma_{12} \partial_{2}-\alpha_{1} \partial_{1} \\
0 & -\partial_{2} & \partial_{1} & 0
\end{array}\right) . \\
& \left(\begin{array}{cccc}
u_{0} & -\alpha_{1} u_{1}+2 \gamma_{12} u_{2} & -\alpha_{2} u_{2} & -\alpha_{1} \alpha_{2} u_{12} \\
u_{1} & u_{0}+2 \gamma_{12} u_{12} & -\alpha_{2} u_{12} & \alpha_{2} u_{2} \\
u_{2} & \alpha_{1} u_{12} & u_{0} & -\alpha_{1} u_{1}+2 \gamma_{12} u_{2} \\
u_{12} & -u_{2} & u_{1} & u_{0}+2 \gamma_{12} u_{12}
\end{array}\right) \\
& =0 .
\end{aligned}
$$




\subsection{Matrix representation in $\mathcal{A}_{n}$}

Using the matrix basis $\beta_{n}$ given in (14), we can get a matrix representation for the Dirac operator in $\mathcal{A}_{n, 2}^{*}$

$$
\mathcal{D}_{n}=\sum_{i=1}^{n} E_{i, n} \partial_{i}
$$

where, $E_{i, n}$ are matrices $\mathbb{R}^{2^{n} \times 2^{n}}$ and $\partial_{i}$ represents the derivative with respect to $x_{i}$.

Let $\Omega$ be an open and connected domain in $\mathbb{R}^{n+1}$ whose points will be denoted by $x=\left(x_{0}, x_{1}, \ldots, x_{n}\right)$. Let, $u(x)$ be a function with values in $\mathcal{A}_{n}$ defined on $\Omega$. Denoting the real-valued components of $u(x)$ by $u_{A}(x)$, that is,

$$
u(x)=\sum_{A} u_{A}(x) E_{A, n},
$$

where $A$ is a set of index combinations and $E_{0, n}=\mathbf{I}_{2^{\mathrm{n}}}$. See [7] for more details on the matrix construction for the operator $\mathcal{D}_{n}$

We can represent the Cauchy-Riemann system as the product of two matrices:

$$
\begin{aligned}
& \mathcal{D}_{n} u(x) \\
& =\mathbf{I}_{2^{\mathrm{n}}} \sum_{A} E_{A, n} \partial_{0} u_{A}(x)+\sum_{i=1}^{n} \sum_{A} E_{i, n} E_{A, n} \partial_{i} u_{A}(x) \\
& =0
\end{aligned}
$$

\subsection{Boundary value problem using fibres}

Theorem 2 Consider a Clifford valued function u given by $u\left(x_{0}, x_{1}, x_{2}\right)=\sum_{A} e_{A} u_{A}\left(x_{0}, x_{1}, x_{2}\right)$, where $e_{A} \in\left\{e_{0}=1, e_{1}, e_{2}, e_{12}\right\}$ such that $u_{A} \in A C^{2}(\Omega)$ Then, the Dirichlet problem in a Decomposition of $\Omega \subset \mathbb{R}^{3}$ in distinguish parts given by:

- The lower covering surface, that is, a distinguishing part for the decomposition in the $x_{0}$-direction representing the 1-dimensional fibre $S_{0}$.

- $S_{01}$, which is the distinguishing part in the $x_{0}, x_{1}$-directions, corresponding to the 2-dimensional fibre.

- $S_{012}$, which is the distinguishing part $x_{0}, x_{1}, x_{2}$-directions, corresponding to the 3-dimensional fibre.

for monogenic function $u$ with values in $\mathbb{R}^{3}$ as defined above, is uniquely solvable. 
Proof: Consider the Cauchy-Riemann operator $\mathcal{D}_{2}$ given by

$$
\begin{aligned}
\mathcal{D}_{2} & =\partial_{0} \mathbf{I}_{4}+\partial_{1} E_{1}+\partial_{2} E_{2} \\
& =\left(\begin{array}{cccc}
\partial_{0} & -\partial_{1} & -\partial_{2} & 0 \\
\partial_{1} & \partial_{0} & 0 & \partial_{2} \\
\partial_{2} & 0 & \partial_{0} & -\partial_{1} \\
0 & -\partial_{2} & \partial_{1} & \partial_{0}
\end{array}\right)
\end{aligned}
$$

Then, the Cauchy-Riemann system $\mathcal{D}_{2} u=0$ for an $\mathcal{A}_{2}$-valued function $u=u_{0} e_{0}+$ $u_{1} e_{1}+u_{2} e_{2}+u_{12} e_{12}$ is given by:

$$
\left(\begin{array}{cccc}
\partial_{0} & -\partial_{1} & -\partial_{2} & 0 \\
\partial_{1} & \partial_{0} & 0 & \partial_{2} \\
\partial_{2} & 0 & \partial_{0} & -\partial_{1} \\
0 & -\partial_{2} & \partial_{1} & \partial_{0}
\end{array}\right)\left(\begin{array}{c}
u_{0} \\
u_{1} \\
u_{2} \\
u_{12}
\end{array}\right)=\mathbf{0}_{4}
$$

By multiplying these matrices, we obtain four equations:

$$
\begin{aligned}
& \partial_{0} u_{0}-\partial_{1} u_{1}-\partial_{2} u_{2}=0 \\
& \partial_{0} u_{1}+\partial_{1} u_{0}+\partial_{2} u_{12}=0 \\
& \partial_{0} u_{2}-\partial_{1} u_{12}+\partial_{2} u_{0}=0 \\
& \partial_{0} u_{12}+\partial_{1} u_{2}-\partial_{2} u_{1}=0
\end{aligned}
$$

First, two of these components have to be prescribed. Without loss of generality, suppose we prescribe arbitrarily $u_{1}$ and $u_{2}$ on the whole boundary $\partial \Omega$, whereas $u_{12}$ has prescribed values on the lower covering surface $S_{0}$ and $u_{0}$ is prescribed at one point of $\bar{\Omega}$. In order to solve the system (17) - (20) we consider the equations (17) (19) as the following system for $u_{0}$ :

$$
\partial_{j} u_{0}=p_{j}, \quad j=0,1,2
$$

where

$$
\begin{gathered}
p_{0}=\partial_{1} u_{1}+\partial_{2} u_{2} \\
p_{1}=-\partial_{0} u_{1}-\partial_{2} u_{12} \\
p_{2}=-\partial_{0} u_{2}+\partial_{1} u_{12}
\end{gathered}
$$

We will show that the right-hand sides of the system (21) are compatible. Consider the expression

$$
\partial_{1} p_{0}-\partial_{0} p_{1}=\partial_{0} \partial_{2} u_{12}+\partial_{0}^{2} u_{1}+\partial_{1} \partial_{2} u_{2}+\partial_{1}^{2} u_{1}
$$

Using the Laplacian for $u_{1}$ and remembering that $\Delta u_{1}=0$ then we can replace $\partial_{0}^{2} u_{1}+\partial_{1}^{2} u_{1}$ by $-\partial_{2}^{2} u_{1}$ in the previous expression. Doing this, we get:

$$
\partial_{1} p_{0}-\partial_{0} p_{1}=-\partial_{2}^{2} u_{1}+\partial_{1} \partial_{2} u_{2}+\partial_{0} \partial_{2} u_{12}=\partial_{2}\left[\partial_{0} u_{12}+\partial_{1} u_{2}-\partial_{2} u_{1}\right]=0
$$


Following from equation (20), the latter expression is zero. Analogously, we use $\Delta u_{2}=0$ to the expression $\partial_{2} p_{0}-\partial_{0} p_{2}$ and use (20) again to conclude that $\partial_{2} p_{0}-$ $\partial_{0} p_{2}=0$. For $\partial_{2} p_{1}-\partial_{1} p_{2}$, we have to use $\Delta u_{12}=0$ to conclude that $\partial_{2} p_{1}-\partial_{1} p_{2}=0$. Thus, the system (21) is compatible and so the component $u_{0}$ is uniquely determined up to an arbitrary constant.

Theorem 3 Consider a Clifford valued function, $u$, given by $u\left(x_{0}, x_{1}, x_{2}, x_{3}\right)=$ $\sum_{A} e_{A} u_{A}\left(x_{0}, x_{1}, x_{2}, x_{3}\right)$, where $e_{A} \in\left\{e_{0}=1, e_{1}, e_{2}, e_{3}, e_{12}, e_{13}, e_{23}, e_{123}\right\}$ such that $u_{A} \in$ $A C^{2}(\Omega)$ Then, knowing the boundary values for the four components $u_{12}, u_{23}$, $u_{123}$ and, $u_{3}$ on the whole boundary, the two components $u_{1}$ and $u_{2}$ can be found from their values on the three-dimensional distinguishing part $S_{1}, S_{2}$ for the boundary, corresponding to the 1-dimensional fibres in the $x_{2}$-direction and, in the $x_{3}$ direction, respectively. Meanwhile, $u_{13}$ can be calculated from the values on the boundary curve $S_{12}$, corresponding to the 2-dimensional fibres in $x_{1}, x_{2}$-directions. The component $u_{0}$, finally, is completely determined by its value at the point $S_{0123}$.

Proof: For this case, we solve the Dirichlet Boundary value problem by prescribing arbitrarily $u_{12}, u_{23}, u_{123}$ and $u_{3}$ on the whole boundary of $\Omega$. The decomposition of $\Omega$ for this case is:

- Two 1-dimensional fibres. One in the $x_{2}$-direction, and the other, in the $x_{3}$-direction.

- One 2-dimensional fibre in the $x_{1}, x_{2}$-directions and

- One 4-dimensional fibre in all directions.

The componentes $u_{1}$ and $u_{2}$ can be found from their values on the 3-dimensional distinguishing part $S_{1}, S_{2}$ for the boundary, corresponding to the 1-dimensional fibres in the $x_{2}$-direction and in the $x_{3}$-direction respectively. Finally, $u_{13}$ can be found from the values on the boundary curve $S_{12}$ corresponding to the 2-dimensional fibres in the $x_{1}, x_{2}$-directions.

The Cauchy-Riemann system $\mathcal{D}_{3} u=0$ for an $\mathcal{A}_{3}$-valued function $u=u_{0} e_{0}+u_{1} e_{1}+$ $u_{2} e_{2}+u_{3} e_{3}+u_{12} e_{12}+u_{13} e_{13}+u_{23} e_{23}+u_{123} e_{123}$ is given by:

$$
\mathcal{D}_{3} u=\left(\begin{array}{c}
\partial_{0} u_{0}-\partial_{1} u_{1}-\partial_{2} u_{2}-\partial_{3} u_{12} \\
\partial_{0} u_{1}+\partial_{1} u_{0}+\partial_{2} u_{3}+\partial_{3} u_{13} \\
\partial_{0} u_{2}-\partial_{1} u_{3}+\partial_{2} u_{0}+\partial_{3} u_{23} \\
\partial_{0} u_{3}+\partial_{1} u_{2}-\partial_{2} u_{1}-\partial_{3} u_{123} \\
\partial_{0} u_{12}-\partial_{1} u_{13}-\partial_{2} u_{23}+\partial_{3} u_{0} \\
\partial_{0} u_{13}+\partial_{1} u_{12}+\partial_{2} u_{123}-\partial_{3} u_{1} \\
\partial_{0} u_{23}-\partial_{1} u_{123}+\partial_{2} u_{12}-\partial_{3} u_{2} \\
\partial_{0} u_{123}+\partial_{1} u_{23}-\partial_{2} u_{13}+\partial_{3} u_{3}
\end{array}\right)=\mathbf{0}_{8}
$$

For the component $u_{0}$, we have the system:

$$
\partial_{i} u_{0}=P_{i}, \quad i=0,1,2,3
$$


where

$$
\begin{gathered}
P_{0}=\partial_{1} u_{1}+\partial_{2} u_{2}+\partial_{3} u_{12} \\
P_{1}=-\partial_{0} u_{1}-\partial_{2} u_{3}-\partial_{3} u_{13} \\
P_{2}=-\partial_{1} u_{2}+\partial_{1} u_{3}-\partial_{3} u_{23} \\
P_{3}=-\partial_{0} u_{12}+\partial_{1} u_{13}+\partial_{2} u_{23}
\end{gathered}
$$

We will show that the right-hand sides of the system (22) are compatible.

Consider the expression

$$
\partial_{3} P_{1}-\partial_{1} P_{3}=\partial_{1} \partial_{0} u_{12}-\partial_{1} \partial_{2} u_{23}-\partial_{1}^{2} u_{13}-\partial_{3} \partial_{0} u_{1}-\partial_{3} \partial_{2} u_{3}-\partial_{3}^{2} u_{13}
$$

Using the Laplacian for $u_{13}$, then we get:

$$
\begin{aligned}
\partial_{3} P_{1}-\partial_{1} P_{3} & =\partial_{0}^{2} u_{13}+\partial_{1} \partial_{0} u_{12}-\partial_{1} \partial_{2} u_{23}+\partial_{2}^{2} u_{13}-\partial_{3} \partial_{0} u_{1}-\partial_{3} \partial_{2} u_{3} \\
& =\partial_{0}\left(\partial_{0} u_{13}+\partial_{1} u_{12}-\partial_{3} u_{1}\right)+\partial_{2}\left(-\partial_{1} u_{23}+\partial_{2} u_{13}-\partial_{3} u_{3}\right)
\end{aligned}
$$

Finally, by rows 6 and 8 from the matrix for $\mathcal{D}_{3} u=0$ we have that $\partial_{3} P_{1}-\partial_{1} P_{3}=0$. Analogously, the remaining equations are equal to zero by using the Laplacian of certain components and certain rows of the matrix $\mathcal{D}_{3} u=0$. We list them here:

- $\partial_{2} P_{1}-\partial_{1} P_{2}=0$ using the Laplacian for $u_{3}$, and rows 4 and 8 from the matrix system.

- $\partial_{2} P_{0}-\partial_{0} P_{2}=0$ using the Laplacian for $u_{2}$, and rows 4 and 7 from the matrix system.

- $\partial_{3} P_{0}-\partial_{0} P_{3}=0$ using the Laplacian for $u_{12}$, and rows 6 and 7 from the matrix system.

- $\partial_{3} P_{2}-\partial_{2} P_{3}=0$ using the Laplacian for $u_{23}$, and rows 7 and 8 from the matrix system.

- $\partial_{1} P_{0}-\partial_{0} P_{1}=0$ using the Laplacian for $u_{1}$, and rows 4 and 6 from the matrix system.

\section{Algorithm 3.1 (System's integrability)}

Input:

- The dimension $n$ of the Clifford algebra $\mathcal{A}_{n}$.

Output:

- The $2^{n-1}$ prescribed components and the 1-dimensional fibres for the system. 


$$
\left(\begin{array}{c}
\mathrm{d} 0 \mathrm{u} 0[x]-\mathrm{d} 1 \mathrm{u} 1[x]-\mathrm{d} 2 \mathrm{u} 2[x] \\
\mathrm{d} 1 \mathrm{u} 0[x]+\mathrm{d} 0 \mathrm{u} 1[x]+\mathrm{d} 2 \mathrm{u} 12[x] \\
\mathrm{d} 2 \mathrm{u} 0[x]-\mathrm{d} 1 \mathrm{u} 12[x]+\mathrm{d} 0 \mathrm{u} 2[x] \\
-\mathrm{d} 2 \mathrm{u} 1[x]+\mathrm{d} 0 \mathrm{u} 12[x]+\mathrm{d} 1 \mathrm{u} 2[x]
\end{array}\right)=\left(\begin{array}{l}
0 \\
0 \\
0 \\
0
\end{array}\right),
$$

where $d j, j=0,1,2$ represent the partial derivative in the respective direction. $D_{2}$ is the $4 \times 4$ matrix, given by:

$$
D_{2}=\left(\begin{array}{cccc}
\mathrm{d} 0 & -\mathrm{d} 1 & -\mathrm{d} 2 & 0 \\
\mathrm{~d} 1 & \mathrm{~d} 0 & 0 & \mathrm{~d} 2 \\
\mathrm{~d} 2 & 0 & \mathrm{~d} 0 & -\mathrm{d} 1 \\
0 & -\mathrm{d} 2 & \mathrm{~d} 1 & \mathrm{~d} 0
\end{array}\right)
$$

and $u$ is given by:

$$
u=\left(\begin{array}{c}
u 0 \\
u 1 \\
u 2 \\
u 12
\end{array}\right)
$$

As we want to obtain solutions for the Cauchy-Riemann system, it is enough to consider $u$ as a $4 \times 1$ matrix. Using the right compatibility condition we get the following explicit result:

$$
\left(\begin{array}{llll}
u_{0} & u_{1} & u_{2} & u_{12}
\end{array}\right)=\left(\begin{array}{llll}
a & x_{1} x_{2} & x_{1} & a-x_{0}
\end{array}\right)
$$

where $a \in \mathbb{R}$.

\section{Initial valued problem for monogenic initial data}

We are interested in solving the initial value problem:

$$
\begin{aligned}
\partial_{t} u & =L u:=\bar{D} u+G(x, u), \\
u(0, x) & =\varphi(x),
\end{aligned}
$$

in the space of monogenic functions satisfying the differential equations

$$
l u:=D u-F(x, u)=0,
$$

where $F(x, u)$ is an anti-monogenic $(\bar{D} f=0)$ function in Clifford Algebras depending on parameters and, the initial function $\varphi$ is a generalized monogenic function satisfying a differential equation with a monogenic right-hand side.

The starting point is the classic Cauchy-Kovalevskaya problem for an evolution equation with a holomorphic right-hand side, where each initial value problem with holomorphic initial data is uniquely solvable. See $[16,21,34]$.

The concept of associated spaces generalizes the connection between monogenic right-hand sides and monogenic initial data. If the initial data belong to an associated space of the right-hand side, then the initial value problem is uniquely solvable. Then, the desired solution of the initial value problem can be found as a fixed point $\left(x^{*}\right)$ of a related operator 


$$
U(x, t)=\varphi\left(x^{*}\right)+\int_{0}^{t} F\left(\tau, x, u, \partial_{j} u\right) d \tau .
$$

In order to construct fixed points for this operator, we have to estimate the integrodifferential operator on the right-hand side of (26). Discussions on the estimate can be found in $[21,23,27,33]$.

On the other hand, let $u=u\left(x_{0}, x_{1}, \ldots, x_{n}\right)$ be the desired solution for a real-valued function with a completely integrable system in $\mu$ variables, $1 \leq \mu \leq 1+n$ :

$$
\partial_{k_{j}} u=p_{k_{j}}, j=1, \ldots, \mu,
$$

where $\partial_{k_{j}}$ means differentiation with respect to the variable $x_{k_{j}}$ in an open and homotopically simply connected subset of the domain under consideration.

Using the concept of a cylindrical domain of order $\mu$ makes it possible to solve $\mu$ dimensional systems (27) not only locally in $\mu$-dimensional subsets; but also, globally in $(1+n)$-dimensional domains, in this case each fibre is defined by a uniquely determined point $x^{*}$ of $S_{k_{1} \ldots k_{\mu}}$. This point is the initial point of the curves $\gamma$ of the particular fibre. Then, an explicit solution can be given by the line integral

$$
U(x)=\varphi\left(x^{*}\right)+\int_{\gamma} \sum_{j=1}^{\mu} p_{k_{j}}(\xi) d \xi_{k_{j}}
$$

where $x^{*}$ is a fixable chosen point and $\gamma$ is any curve connecting $x^{*}$ and $x$ (in the subset). The integral does not depend on the special choice of $\gamma$ because the system is completely integrable and the subset is homotopically simply connected by hypothesis. Finally, in each fibre the values of $u$ are given by an integral (28) where $\gamma$ is located in the corresponding fibre. More information related to the initial value problems can be found in $[1,10,14,15,32]$.

\section{References}

[1] E. Ariza, A. Di Teodoro, M. Sapian and F.Vargas Sufficient Conditions for First Order Differential Operators to be Associated with a q-Metamonogenic Operator in a Clifford Type Algebra; CMFT Computational Methods and Function Theory, vol 17, 2, pp 211-236, (2017).

[2] H. Begehr, Boundary value problems in complex analysis I and II, Boletín de la Asociación Matemática Venezolana, Vol.XII, Nro 1-2, 65-85, 217-250, (2005).

[3] H. Begehr, Integral representations in complex, hypercomplex and Clifford analysis, Integral transform and Special Functions 13, 223-241 (2002).

[4] C. Balderrama, A. Di Teodoro and A. Infante, Some Integral Representation for Meta-Monogenic Function in Clifford Algebras Depending on Parameters. Adv. Appl. Clifford Algebras, 23, 4, 793-813, (2013).

[5] W. Baylis Clifford (Geometric) Algebras With Applications in Physics, Mathematics, and Engineering Birkhäuser; 1st ed. 1996. Corr. 2nd printing 1999 edition (April 23, 1999). 
[6] F. Brackx, R. Delanghe and F. Sommen, Clifford analysis, Pitman Research Notes, vol. 76, (1982).

[7] J. Ceballos, A. Di Teodoro and F. Vargas. Matrix approach to Clifford-type algebra: A computational implementation. Submitted 2018.

[8] R. Delanghe, F. Sommen and V. Soucek Clifford Algebra and Spinor-Valued Functions: A Function Theory for the Dirac Operator (Mathematics and Its Applications), Springer, vol. 53, (1992).

[9] A. Di Teodoro, R. Franquiz and A. Lopez, A modified Dirac Operator in Parameter-Dependent Clifford Algebra: A Physical Realization, Adv. Appl. Clifford Algebras. vol. 25, issue 2, pp 303-320, (2015).

[10] A. Di Teodoro \& Maria Sapiain Solution of the initial value problem for no linear differential equation in general Clifford type. Advances in Applied Clifford Algebras. vol. 25, 2, pp 283-301, (2015).

[11] A. Di Teodoro and C.J. Vanegas. A Dirichlet Problem for the First Order Inhomogeneous Meta-Monogenic Equation in Parameter Depending Clifford Algebras. Complex Analysis and Operator Theory vol. 9, 5, 1025-1036, (2015).

[12] R. Feynman, R. Leighton and M. Sands, The Feynman Lectures on Physics: Definitive and Extended Edition, Addison Wesley, (2005).

[13] F. John, Partial differential equations, Springer-Verlag. Berlin and New York, (1971).

[14] L. H. Son and N. Q. Hung, The initial value problems in Clifford and quaternion analysis. Proceedings of the 15th ICFIDCAA 2007, Osaka Municipal Universities Press, 3, 317-323, (2008).

[15] L. H. Son and W. Tutschke, First order differential operator associated to the Cauchy-Riemann Operatorin the Plane, Complex Variables., Vol. 48, 797-801, (2003).

[16] L. H. Son and W. Tutschke. Complex methods in higher dimensions, recent trends for solving boundary value and initial value problems. Complex Variables, vol. 50 (No. 7-11), pp. 673-679 (2005).

[17] L.H. Son, W. Tutschke and S. Jain (editores), Methods of Complex and Clifford Analysis, Proceedings of the International Conference on Applied Mathematics based on partial differential equations and complex analysis (ICAM), Hanoi August 25-29, 2004, SAS International Publications (2006).

[18] E. Obolashvili, Partial Differential Equations in Clifford Analysis, 1 ed., Chapman and Hall/CRC, (1999).

[19] E. Obolashvili, Higher Order Partial Differential Equations in Clifford Analysis Effective solutions, Birkhäuser, Boston, (2003).

[20] Peres N.M.R., Scattering in one-dimensional heterostructures described by the Dirac equation J. Phys.: Condens. Matter 21, 095501, (2009).

[21] W. Tutschke. Solution if initial value problems in classes of generalized analytic functions. Teubner Leipzig and Springer-Verlag, (1989).

[22] W. Tutschke, An elementary approach to Clifford analysis. Contained in the Proceedings [ Mshimba A.S.A., and Tutschke W., (eds.), Functional Analytic Methods in Complex Analysis and Applications to Partial Differential Equations. Proceedings of the Second Workshop held at the ICTP in Trieste, January 25-29. World Scientific (1995).], pp. 402-408. 
[23] W. Tutschke, Interior estimates in the theory of partial differential equations and their applications to initial value problems, contained in Mem. Diff. Equat. Math. Phys. Vol. 12, 204-209, (1997).

[24] W. Tutschke, Real and complex fundamental solutions. A way for unifying mathematical analysis. Bol. Asoc. Mat. Venez., vol. 9, no. 2, pp. 141-179, (2002).

[25] W. Tutschke, Complex analysis within the framework of analytical methods for partial differential equations. Contained in the collection of papers [17], pp. 123-140, (2006).

[26] W. Tutschke. The Distinguishing Boundary for Monogenic Functions of Clifford Analysis. Adv. Appl. Clifford Algebras, vol. 25, 2, 441-451, (2015).

[27] W. Tutschke and N. T. Van, Interior Estimates in the sup-norm for Generalized Monogenic Functions Satisfying a Differential Equation with Anti-Monogenic Right-Hand Sides, Complex Variable and Elliptic Equation, vol. 52, 5, 367375, (2007).

[28] W. Tutschke and C. J. Vanegas, Clifford algebras depending on parameters and their applications to partial differential equations, Contained in Some topics on value distribution and differentiability in complex and p-adic analysis. Beijing: Science Press. Mathematics Monograph Series 11, 430-450, (2008).

[29] W. Tutschke and C. J. Vanegas, Métodos del análisis complejo en dimensiones superiores. Ediciones IVIC, Caracas, (2008).

[30] W. Tutschke and C. J. Vanegas, A boundary value problem for monogenic functions in parameter-depending Clifford algebras. Complex Variables and Elliptic Equations, vol. 56, issue 1-4, 113-118, (2011).

[31] W. Tutschke and H. L. Vasudeva, An Introduction to Complex Analysis. Classical and Modern Approaches, Chapman \& Hall/CRC, (2005).

[32] W. Tutschke and U. Yüksel, Generalized Monogenic Functions Satisfying Differential Equations with Anti-Monogenic Right-Hand Sides, ISAAC series, Kluwer Academic Publishers, Dordrecht, 6, 263-270, (1999).

[33] W. Tutschke and U. Yüksel, Interrior Lp-estimates for Functions with Integral Representations, Appl. Anal, 73 , 281-294, (1999).

[34] U. Yüksel and O. Çelebi, Solution of Initial Value Problems of CauchyKovalevsky Type in the Space of Generalized Monogenic Functions, Adv. Appl. Clifford Algebras., Vol. 20, Issue 2, 427-444, (2010). 